\title{
Macrosteatosis and microsteatosis respond differently to intermittent hepatic inflow occlusion
}

New findings suggest that liver transplant donors who have macrosteatosis are less tolerant of hepatic ischaemia-reperfusion injury (IRI) associated with intermittent hepatic inflow occlusion (IHIO) than donors who have microsteatosis.

Previous work has demonstrated that IHIO is a safe and effective method to reduce blood loss in living donor hepatectomy. However, whether this technique is safe in donors with liver

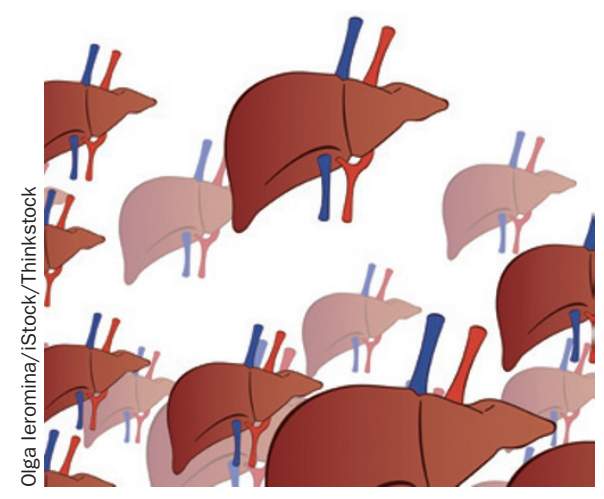

steatosis-either macrosteatosis or microsteatosis-is unclear. As a result, Sangbin Han and colleagues compared the tolerance to hepatic IRI in donors with macrosteatosis and/or microsteatosis.

The study included 144 donors who underwent a right hepatectomy. Participants had macrosteatosis, microsteatosis, a combination of both, or no steatosis. The donors were divided into two groups for comparison; non-macrosteatosis $(n=68)$ versus macrosteatosis $(n=76)$ and non-microsteatosis $(n=51)$ versus microsteatosis $(n=93)$. The researchers used the coefficient of the regression line between the cumulative IHIO time and the peak concentrations of aspartate aminotransferase and alanine aminotransferase after surgery as a surrogate marker for tolerance to hepatic IRI.

The coefficient was significantly greater in the macrosteatosis group compared with the non-macrosteatosis group. However, the coefficient was comparable between donors with or without microsteatosis. In the subgroup of patients who underwent $>30$ min of IHIO, aminotransferase levels were higher in donors with macrosteatosis than in those with microsteatosis, and biliary complications were increased in donors with macrosteatosis.

"Microsteatosis has a limited impact on the tolerance of hepatic IRI, whereas macrosteatosis significantly impaired the liver tolerance," conclude Han and co-workers. The team hope their findings will help expand the donor pool and improve outcomes for donors and recipients.

\section{Claire Greenhill}

Original article Han, S. et al. Comparison of the tolerance of hepatic ischemia reperfusion injury in living donors: macrosteatosis versus microsteatosis. Liver Transplant. doi:10.1002/It.23878 